\section{ENERGY REQUIREMENTS OF INDIAN ATHLETES IN VARIOUS SPORTS}

Y Venkata Ramana Assistant Director, Department of Work Physiology and Sports Nutrition, National Institute of Nutrition (ICMR), Hyderabad, Andhra Pradesh, India

10.1136/bjsm.2010.078725.132

Success in sports depends mainly on three factors, the genetic endowments, the state of training and nutrition. Nutrition has always been of great interest to athletes. Proper nutrition is an important component of the total training programme. Athletes need the same amount of essential nutrients as nonathletes with varied increases in their caloric requirements as well as other macro and micronutrients due to increased physical activity. Therefore, it is essential to explore and assess these increased demands of macro and micronutrients, especially the energy to support their training before, during and after the competition for achieving desirable body weight and composition and optimal sports performance. All sporting events involve some degree of intense muscular training, which depend on the type of sport and phase of training. Hence, the nutritional needs, especially the energy requirements, differ greatly from athlete to athlete. The energy requirements of an athlete is also influenced by the body size and composition, age and the gender apart from the type of sport, different phases of training and non-training activities. As a result, a wide range of energy intake levels is observed among athletes when considering within and between the sporting events. Therefore, it is essential to consider these factors when recommending energy allowances apart from dietary preferences of athletes for achieving desirable body weight, composition and optimal sports performance. The research findings in this direction and recommended energy allowances for Indian athletes along with the scope for further research in India will be discussed. 